\title{
Achieving the Preperitoneal Space in Totally Extraperitoneal Inguinal Hernia Repair: Dissection with or without a Balloon Dissector
}

\author{
Ah Young Kang, M.D., Sung Ryol Lee, M.D., Byung Ho Son, M.D., Ph.D., Kyung Uk Jung, M.D. \\ Department of Surgery, Kangbuk Samsung Hospital, \\ Sungkyunkwan University School of Medicine, Seoul, Korea
}

\begin{abstract}
Purpose: A balloon dissector is widely used to achieve the preperitoneal space in totally extraperitoneal (TEP) inguinal hernia repair. The aim of this study was to compare the operative results of TEP cases performed with (the balloon dissection group) or without (the plain dissection group) a balloon dissector.

Methods: A retrospective analysis was conducted with a consecutive series of inguinal hernia repairs performed by a single surgeon in 000 Hospital between April 2008 and April 2012. All 128 patients with full-length video recordings were included. The distribution of the operation method was altered during the study period, from dissection with a balloon dissector to without it.
\end{abstract}

Results: Of 128 cases, 57 belonged to the balloon dissection group and the other 71 belonged to the plain dissection group. The demographic features and clinical characteristics were similar in both groups. Mean operation time ( 57.7 vs. 45.6 $\min , p<0.001)$ and laparoscopic recording time (31.6 vs. 25.0 $\min , p=0.004$ ) were significantly shorter in the plain dissection group without differences in the degree of bloodstaining and the frequency of peritoneal tearing. Postoperative complications did not differ between the two groups.

Conclusion: Plain dissection may be a safe and feasible alternative method of achieving the preperitoneal space in TEP by an experienced surgeon.

Key words: TEP, Balloon dissection, Spacemaker

\section{INTRODUCTION}

Laparoscopic inguinal hernia repair emerged in the 1990s, and it now accounts for $15 \sim 20 \%$ of hernia operations worldwide. ${ }^{1}$ Among several types of laparoscopic inguinal hernia repair, totally extraperitoneal (TEP) inguinal hernia repair is the preferred procedure. ${ }^{2}$ The major advantage of TEP is non-violation of the peritoneal cavity, which minimizes the risk of clinically significant adhesions or visceral organ injury. Although it is a technically difficult procedure with a long learning curve because of space constraints and unfamiliar anatomy. ${ }^{3}$ In this respect, balloon dissector can provide significant assistance to a surgeon to perform an easier and more consistent extraperitoneal dissection, especially during the learning curve. ${ }^{4-6}$ However, use of a balloon dissector does not guarantee the safe dissection without adjacent tissue injuries at all times. It can also cause the tear of vessels or peritoneum, injure the bowel or

Received July 8, 2014, Revised 1st, September 9, 2014; 2nd, October 1, 2014; 3rd, October 13, 2014, Accepted October 15, 2014

※ Corresponding author: Kyung Uk Jung

Department of Surgery, Kangbuk Samsung Hospital Samsung Medical Center, Sungkyunkwan University School of Medicine, 29, Saemunan-ro, Jongno-gu, Seoul 110-746, Korea

Tel : +82-2-2001-8576, Fax : +82-2-2001-8360

E-mail : sahelgrean@gmail.com bladder, particularly in patients who have undergone previous lower abdominal surgery. ${ }^{5}$ On the other hand, meticulous dissection to create the preperitoneal space under the magnified view of a laparoscope is not as difficult for an experienced surgeon with technical competence in this procedure.

The aim of this study was to compare the operative results including the quality of laparoscopic visual field in TEP cases that were performed with (the balloon dissection group) or without (the plain dissection group) a balloon dissector.

\section{MATERIALS AND METHODS}

\section{1) Patients and data collection}

This is a retrospective analysis of a consecutive series of inguinal hernia repairs performed by a single surgeon in $\mathrm{OOO}$ Hospital between April 2008 and April 2012. The surgeon started laparoscopic inguinal hernia repair in 2007 and has performed more than 60 cases every year. In early days, a balloon dissector was used in all cases. From 2010, the preperitoneal space was made without a balloon dissector and this technique gradually replaced the original method.

During the study period, a total of 363 patients underwent any kind of operative procedure for unilateral inguinal hernia repair and 274 of them were laparoscopic TEP. Of these 274 cases, full-length video recordings were available for 128 
patients. Because we wanted to analyze the visual field of the operative procedure by making use of video recordings, only these 128 patients were included in this study. Patient demographics, operative details including the grade of bloodstaining in the visual field before mesh application, and postoperative outcomes were analyzed. The operation time was defined as the time from incision to skin closure. The recording time was the time from initial visualization of the subcutaneous tissue with the laparoscope to the removal of the scope. The sac time was the time from initial visualization of the subcutaneous tissue to the first grasp of the hernia sac. The number of cautery uses was defined as the number of times that the electrocautery device was used until we found the hernia sac. This included all the cauterizations used for dissection and bleeding control. The degree of bloodstaining was graded by an independent observer, an experienced general surgeon who did not participate in the surgical procedure. At the time when the surgeon grabbed the hernia sac or shortly before then, when the hernia sac was in the center of the screen, the observer assessed the amount of bloodstaining and graded it semi-quantitatively as follows: Grade I, less than a quarter of the tissue in the screen stained with blood; Grade II, less than half of the tissue stained; Grade III, less than three-quarters of the tissue stained; and Grade IV, more than three-quarters of the tissue stained (Fig. 1).

We assessed postoperative pain, scrotal swelling and urinary retention in the immediate postoperative period. Hospital stay was also analyzed. Sustained pain, operation site swelling, seroma in the inguinal area, hydrocele and wound infections were assessed in the outpatient clinic, at the first visit. Sustained pain was defined as operative site pain that required any kind of painkiller at the one-week outpatient follow-up. Operative site swelling was diagnosed by simple inspection and palpation.
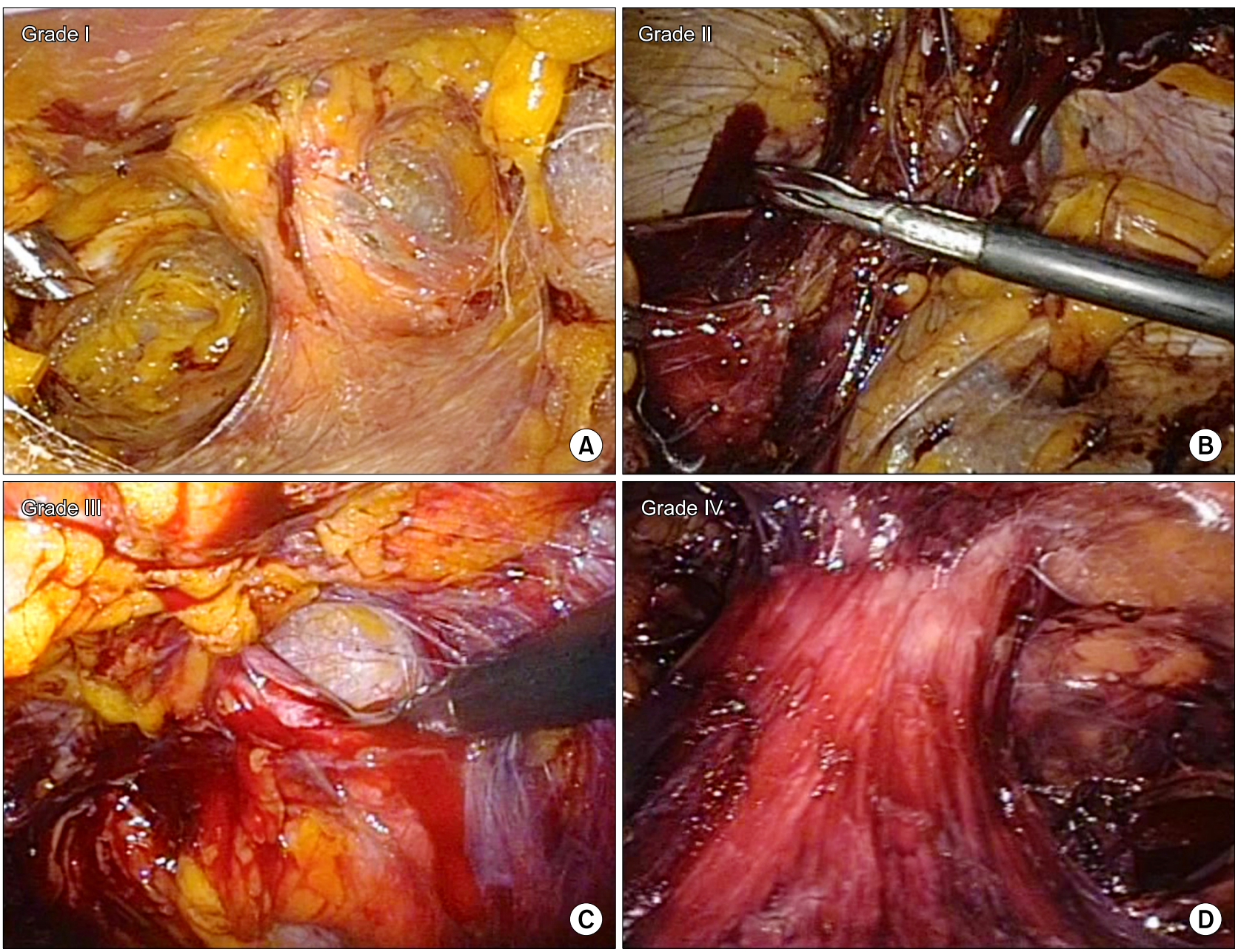

Fig. 1. Semiquantitative grading system of the bloodstain. (A) Grade I: Grade I, less than a quarter of the tissue in the screen stained with blood, (B) Grade II: less than half of the tissue stained, (C) Grade III: less than three quarters of the tissue stained; (D) Grade IV: more than three quarters of tissue stained. 
Seroma in the inguinal area was defined as a case in which the volume of aspirated fluid was greater than $5 \mathrm{~mL}$. Hydrocele was diagnosed using an ultrasonography.

\section{2) Surgical technique}

Laparoscopic TEP was performed using a two- or three-port technique. After creating a channel between the rectus muscle and the posterior sheath, the preperitoneal space was created by using a balloon trocar (Spacemaker, Autosuture, Norwalk, CT, USA) (the balloon dissection group) or without one (the plain dissection group). We have described the detailed procedure of each technique in the balloon dissection group in a previous report. ${ }^{7}$ In the plain dissection group, most of the cases were performed with a two-port technique. After insertion of a wound retractor and a custom-made glove port consisting of a surgical glove and two $5 \mathrm{~mm}$ trocars or a LapSingle ${ }^{\mathrm{TM}}$ (Sejong Medical Co., Ltd., Paju, Korea), meticulous dissection was started from a small space between the rectus muscle and the posterior sheath. With $\mathrm{CO}_{2}$ gas insufflation, soft tissue looking like a spider web was dissected with an endodissector and endoscissors, and the small vessels were cauterized with monopolar electrocautery. Other surgical steps, apart from the way in which the preperitoneal space was achieved, were identical with those of the balloon dissection group. After identification of Cooper's ligament and Bogros' space, the hernia sac was assessed and ligated using black silk. We used Parietex ${ }^{\mathrm{TM}}$ Anatomical Mesh (Covidien, Mansfield, MA, USA) $13 \times 9 \mathrm{~cm}$ in size. The mesh was applied after making a suitable cut in each patient. Then it was fixed to the vicinity of the inferior epigastric artery using one or two tackers (ProTack ${ }^{\mathrm{TM}}$, Autosuture), and to the vicinity of the Cooper's ligament using two milliliters of Tissel (Baxter AG, Vienna, Austria).

\section{3) Statistics}

Data analysis was performed using SPSS software version 18.0 (IBM SPSS Statistics, Armonk, NY, USA). Data were analyzed using the chi-squared test and Student's $t$-test. Results were regarded as significant when $p<0.05$.

\section{RESULTS}

Of the 128 patients, whose full-length video recordings were available, 57 were in the balloon dissection group and 71 were in the plain dissection group. Most of the patients were male (93.8\%) and the proportion of indirect hernia was higher $(60.9 \%)$ than the other types. The gender distribution, age, body mass index (BMI), American Society of Anesthesiologists (ASA) score, type and direction of inguinal hernia were not sig-

Table 1. Demographic and clinical characteristics of the patients

\begin{tabular}{|c|c|c|c|}
\hline & $\begin{array}{l}\text { Balloon dissection } \\
\qquad(\mathrm{N}=57)\end{array}$ & $\begin{array}{l}\text { Plain dissection } \\
\qquad(\mathrm{N}=71)\end{array}$ & $p$ value \\
\hline Gender (male) & $56(98)$ & $64(90)$ & 0.08 \\
\hline Age $(y r)$ & $59.4 \pm 16.7$ & $61.4 \pm 17.1$ & 0.51 \\
\hline BMI $\left(\mathrm{kg} / \mathrm{m}^{2}\right)$ & $24.0 \pm 2.9$ & $23.5 \pm 2.4$ & 0.31 \\
\hline ASA score & & & 0.65 \\
\hline I & $24(42)$ & $28(39)$ & \\
\hline II & $28(49)$ & $33(47)$ & \\
\hline III & $5(9)$ & $10(14)$ & \\
\hline Type of hernia & & & 1.00 \\
\hline Indirect & $50(88)$ & $62(87)$ & \\
\hline Direct & $3(5)$ & $4(6)$ & \\
\hline Combined & $4(7)$ & $5(7)$ & \\
\hline Direction of hernia & & & 0.70 \\
\hline Right & $35(61)$ & $43(61)$ & \\
\hline Left & $21(37)$ & $28(39)$ & \\
\hline Bilateral & $1(2)$ & $0(0)$ & \\
\hline Use of antiplatelet agent & $5(9)$ & $5(7)$ & 0.75 \\
\hline Previous abdominal OP history & $7(12)$ & $12(17)$ & 0.47 \\
\hline Previous low abdominal OP history & $4(7)$ & $8(11)$ & 0.54 \\
\hline Previous inguinal hernia history & $5(9)$ & $5(7)$ & 0.75 \\
\hline
\end{tabular}

Data are presented as the number $(\%)$ or the mean \pm SD. OP $=$ Operation. 
nificantly different between the two groups (Table 1). Perioperative use of an antiplatelet agent, history of a previous abdominal and low abdominal operation, and history of inguinal hernia repair were also not significantly different between the two groups.

Table 2 shows the operative details of the patients. In the plain dissection group, a two-port technique was used more frequently ( $75 \%$ vs. $96 \%, p=0.001)$. Mean operation time was shorter in the plain dissection group (57.7 vs. $45.6 \mathrm{~min}, p<$ 0.001 ). Mean recording time was also shorter in the plain dissection group (31.6 vs. $25.0 \mathrm{~min}, p=0.004$ ). As expected, we used the electrocautery device more frequently in the plain dis-

Table 2. Operative details of the patients

\begin{tabular}{lccc}
\hline & $\begin{array}{c}\text { Balloon } \\
\text { dissection } \\
(\mathrm{N}=57)\end{array}$ & $\begin{array}{c}\text { Plain } \\
\text { dissection } \\
(\mathrm{N}=71)\end{array}$ & $p$ value \\
& $43(75)$ & $68(96)$ & 0.001 \\
\hline Two ports technique & $57.7 \pm 20.4$ & $45.6 \pm 12.0$ & $<0.001$ \\
Operation time (min) & $31.6 \pm 13.3$ & $25.0 \pm 7.7$ & 0.004 \\
Recording time (min) & $7.8 \pm 3.8$ & $8.2 \pm 3.2$ & 0.22 \\
Sac time (min) & $1.1 \pm 2.5$ & $2.7 \pm 2.1$ & $<0.001$ \\
Number of cautery use & & & 0.46 \\
Degree of bloodstain & $24(42)$ & $35(49)$ & \\
$\quad$ I & $18(32)$ & $19(27)$ & \\
II & $9(16)$ & $14(20)$ & \\
III & $6(11)$ & $3(4)$ & \\
IV & $12(21)$ & $14(20)$ & 0.85 \\
Tear in peritoneum &
\end{tabular}

Data are presented as the number $(\%)$ or the mean \pm SD. section group $(p<0.001)$. However, the sac time, the time to first grasp of the hernia sac, and the degree of bloodstaining were not different between two groups. The frequency of peritoneal tearing was similar in both groups. There were no conversions or intraoperative complications. No bladder, bowel or epigastric vessel injury was detected during the procedures.

Table 3 indicates the length of total hospital stay of the patients, postoperative pain and complications. Immediate scrotal swelling and postoperative pain were not different between two groups. There was no difference in complications including urinary retention, sustained pain, operative site swelling, seroma in the inguinal area, hydrocele and wound infection between the two groups.

\section{DISCUSSION}

Laparoscopic surgery, which was designed to reduce the surgical stress and complications associated with large incisions, has been shown to improve short-term outcomes without compromising long-term results and is gaining popularity in various kinds of surgical procedures. Since Ferzli et al. reported the first TEP inguinal hernia repair in $1992,{ }^{8}$ it has also proven to be safe and effective and TEP has been accepted as a standard alternative to the conventional open procedure.

Although it has been associated with well-known advantages of laparoscopic surgery such as less pain and faster recovery, ${ }^{9-11}$ the widespread dissemination of TEP has been hampered by technical difficulties and a steep learning curve. ${ }^{3,12}$ As the working space available in TEP is very restricted especially in the

Table 3. Postoperative outcomes of the patients

\begin{tabular}{lccc}
\hline & $\begin{array}{c}\text { Balloon dissection } \\
(\mathrm{N}=57)\end{array}$ & $\begin{array}{c}\text { Plain dissection } \\
(\mathrm{N}=71)\end{array}$ & 0.90 \\
\hline Hospital stay (days) & $4.2 \pm 2.1$ & $4.1 \pm 5.6$ & 0.20 \\
Immediate postoperative scrotal swelling & $2(3.5)$ & $0(0)$ & 0.92 \\
Immediate postoperative pain, VAS $\geq 5$ & & & 0.48 \\
Immediate postoperative & $18(32)$ & $23(32)$ & 0.69 \\
$>$ arrival at ward, $\leq 1$ hour & $7(12)$ & $6(9)$ & 1.00 \\
$>1$ hour, $\leq 4$ hours & $2(4)$ & $4(6)$ & 1.00 \\
Urinary retention & $3(5)$ & $4(6)$ & 0.20 \\
Outpatient clinic, first visit & $1(1)$ & $12(2)$ & 0.44 \\
$\quad$ Sustained pain & $5(9)$ & $0(0)$ & 0.44 \\
Operation site swelling & $1(2)$ & $0(0)$ & 0.50 \\
$\quad$ Seroma in the inguinal area & $1(2)$ & $2(3)$ & \\
Hydrocele & $0(0)$ & & \\
Wound infection & & & \\
\hline
\end{tabular}

Data are presented as the number $(\%)$ or the mean \pm SD. VAS $=$ Visual Analogue Scale. 

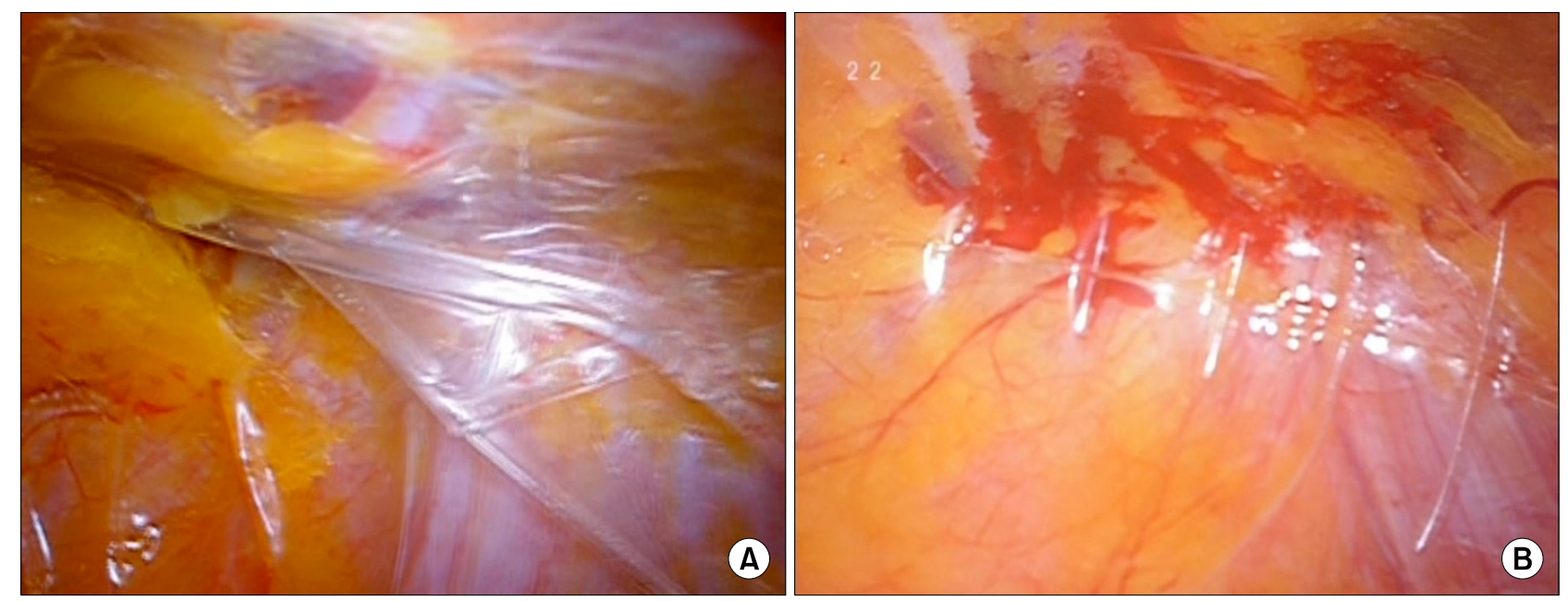

Fig. 2. View of preperitoneal space under blunt dissection. (A) View from inside of the balloon dissector. (B) Tear of small vessels and bleeding induced by blunt injury.

initial stages of dissection, surgeons have generally used balloon dissectors to achieve the preperitoneal space. ${ }^{4-7}$ However, care should be taken even when a balloon dissector is used, because the possibility of a peritoneal tear, injury of adjacent organs, and bleeding, which compromises the visual field and disturbs the identifications of important anatomical structures, still exists (Fig. 2). ${ }^{5}$ When the balloon is inflated, almost all of the soft tissues and minor vessels are compressed by the balloon; it crushes the surrounding tissue indiscriminately, and the soft tissue fragments and oozing from the minor vessels can obscure the operative field.

On the other hand, after understanding the laparoscopic anatomy of the preperitoneal space, if the surgeon is highly experienced and knows the right direction to the symphysis pubis and Cooper's ligament, it is not as difficult to make sufficient space without a balloon dissector by performing meticulous dissection under direct visualization of the surrounding soft tissues.

There have been a few studies comparing the balloon dissection group with the plain dissection group in TEP, which resulted in conflicting conclusions. ${ }^{13-15}$ While Mirsa et al. reported reduced postoperative pain, scrotal edema and seroma formation in the balloon dissection group compared to the plain dissection group, ${ }^{13}$ Bringman et al. insisted that the dissection with a balloon dissector was not superior to the telescopic plain dissection. $^{15}$

In this study, we expected a lower degree of bloodstaining in the plain dissection group before analyzing the data, which may result in better vision during the operation, a shorter operation time, and fewer complications. We were discouraged to find that although the proportion of Grade I patients was slight- ly higher in the plain dissection group, the degree of bloodstaining was not statistically different between the two groups. However, the operation time and the recording time were significantly shorter in the plain dissection group without an increase in postoperative complications. These statistical differences remained after stratification of the patients with age ( $<60$ vs. $\geq 60$ ), BMI ( $<25.0$ vs. $\geq 25.0$ ), and ASA score (I+II vs. III+IV) (data not shown). Plain dissection without a balloon dissector appeared to be a safe and feasible technique to achieve the preperitoneal space in TEP and might be even faster than using a balloon dissector in experienced hands. However, these differences were not maintained in the group of patients who used an antiplatelet agent during the operation, or who had a history of abdominal operation, or inguinal hernia repair (data not shown). It may be that such operations are so complicated in these groups of patients that these study parameters were less influenced by the surgical technique.

There were some limitations in this study. Because of its retrospective nature, we could not exclude the possibility of bias, the surgeon factor. The distribution of the operation method was altered during the study period. While the surgeon originally used the balloon dissector in all cases, most of the surgeries were performed without it in the later periods of the study. Similar to other laparoscopic surgeries, the technique of hernia repair generally improves over time, as experience increases, and the proficiency is strongly related to the operation time and complication rate. Therefore, we cannot completely exclude the possibility that the different periods of operating using each technique influenced the results of the study. In addition, we only assessed immediate postoperative and short- 
term complications. To draw a proper interpretation and generative conclusive evidence, a prospective randomized trial with long-term follow-up should be conducted.

\section{CONCLUSION}

In conclusion, a balloon dissector is a helpful but not always necessary instrument for creating the preperitoneal space. The experienced surgeon may make the preperitoneal space safely without a balloon dissector.

\section{REFERENCES}

1) Davis CJ, Arregui ME. Laparoscopic repair for groin hernias. Surg Clin North Am 2003;83:1141-1161.

2) McCormack K, Scott NW, Go PM, Ross S, Grant AM. EU Hernia Trialists Collaboration. Laparoscopic techniques versus open techniques for inguinal hernia repair. Cochrane Database Syst Rev 2003:Cd001785.

3) Brandt-Kerkhof A, van Mierlo M, Schep N, Renken N, Stassen L. Follow-up period of 13 years after endoscopic total extraperitoneal repair of inguinal hernias: a cohort study. Surg Endosc 2011;25:1624-1629.

4) Voeller GR, Mangiante EC, Jr. Totally preperitoneal laparoscopic inguinal herniorrhaphy using balloon distention. Scand J Gastroenterol Suppl 1995;208:67-73.

5) Putnis $S$, Berney CR. Totally extraperitoneal repair of inguinal hernia: techniques and pitfalls of a challenging procedure. Langenbecks Arch Surg 2012;397:1343-1351.

6) Schwaitzberg SD, Hermann GD. Transballoon trocar insertion during preperitoneal hernia repair. Surg Endosc 2001;15:12351236.

7) Kwon KH, Son BH, Han WK. Laparoscopic totally extraperi- toneal repair without suprapubic port: comparison with conventional totally extraperitoneal repair. J Korean Surg Soc 2011;80:319-126.

8) Ferzli GS, Massad A, Albert P. Extraperitoneal endoscopic inguinal hernia repair. J Laparoendosc Surg 1992;2:281-286.

9) Chung RS, Rowland DY. Meta-analyses of randomized controlled trials of laparoscopic vs conventional inguinal hernia repairs. Surg Endosc 1999;13:689-694.

10) Choi YY, Han SW, Bae SH, Kim SY, Hur KY, Kang GH. Comparison of the outcomes between laparoscopic totally extraperitoneal repair and prolene hernia system for inguinal hernia; review of one surgeon's experience. J Korean Surg Soc 2012;82:40-44.

11) Dahlstrand U, Sandblom G, Ljungdahl M, Wollert S, Gunnarsson U. TEP under general anesthesia is superior to Lichtenstein under local anesthesia in terms of pain 6 weeks after surgery: results from a randomized clinical trial. Surg Endosc 2013;27:3632-3638.

12) Lau H, Patil NG, Yuen WK, Lee F. Learning curve for unilateral endoscopic totally extraperitoneal (TEP) inguinal hernioplasty. Surg Endosc 2002;16:1724-1728.

13) Mirsa MC, Kumar S, Bansal VK. Total extraperitoneal (TEP) mesh repair of inguinal hernia in the developing world: comparison of low-cost indigenous balloon dissection versus direct telescopic dissection: a prospective randomized controlled study. Surg Endosc 2008; 22: 1947-1958.

14) Bringman S, Ek A, Haglind E, Heikkinen TJ, Kald A, Kylberg $\mathrm{F}$, et al. Is a dissection balloon beneficial in totally extraperitoneal endoscopic hernioplasty (TEP)? A randomized prospective multicenter study. Surg Endosc 2001;15:266-270.

15) Bringman S, Ek A, Haglind E, Heikkinen TJ, Kald A, Kylberg $\mathrm{F}$, et al. Is a dissection balloon beneficial in bilateral, totally extraperitoneal, endoscopic hernioplasty? A randomized, prospective, multicenter study. Surg Laparosc Endosc Percutan Tech 2001;11:322-326. 